\title{
Color Medical Image Enhancement Based on Adaptive Equalization of Intensity Numbers Matrix Histogram
}

\author{
Ju-Ping Gu Liang Hua Xiao Wu Hui Yang Zhou Zhou \\ Department of Electrical Engineering, Nantong University, Nantong 226019, China
}

\begin{abstract}
The enhancement technique for color medical images is conductive to improve the resolution and accuracy of the original image. A new enhancement method combining the Young-Helmholtz (Y-H) transformation with the adaptive equalization of intensity numbers matrix histogram is proposed in this paper. The adaptive histogram equalization method is applied to strengthen the details, enhance the contrast, and suppress the noise of the original image effectively. The enhanced image can be displayed in the red-greenblue (RGB) color space through inverse Y-H transformation with the same hue and saturation. The experiment results demonstrate that the method has the enhancement effect with low computational complexity, which provides the foundation for the medical diagnosis and further processing of medical images.
\end{abstract}

Keywords: Youag-Helmhotz (Y-H) color space, adaptive histogram equalization, medical image enhancement, image processing, color space transformation.

\section{Introduction}

The enhanced technique is widely used for color medical images to achieve the improvement of visual effect and resolution, which makes it more helpful for the computer processing. According to different types of images, the enhancement methods can be divided into gray image enhancement and color image enhancement. Depending on the enhancement of the space, the enhanced methods can be divided into spatial domain and frequency domain enhancement. Most of the traditional enhanced methods based on contrasting are always restricted by the setting of parameters and specific applications. During the color image processing, color distortion happens frequently since the primary three colors named red, green and blue are highly correlated. In order to avoid the image distortion, many methods have been tried to convert the image to a certain image with a separated chrominance and luminance color space. Blottal et al. ${ }^{[1]}$ realized the enhancement of color medical pathology images which were captured by electronic microscope and endoscope in the hue-saturation-intensity (HSI) space. Yu et al. ${ }^{[2]}$ adopted the pretreatment with gray stretch method and the histogram equalization algorithm with the adaptive histogram to enhance the color image from endoscope in white brightness-color brightness-hue

\footnotetext{
Regular paper

Manuscript received September 30, 2013; accepted May 5, 2014

This work was supported by National Natural Science Foundation of China (Nos. 61273024 and 61305031), the Infrastructure Construction Foundation of Science and Technology in Jiangsu Province (No. BM2011089), Natural Science Fund of Jiangsu Province and 333 fund (Nos. 12KJB510023 and KB2012227) and the Priority Academic Program Development of Jiangsu Higher Education Institutions (PAPD).

Recommended by Guest Editor Xin Sun

(C) Institute of Automation, Chinese Academy of Science and Springer-Verlag Berlin Heidelberg 2015
}

(WCH) space. Considering the complexity of converting the RGB space into HSI space. Wang et al. ${ }^{[3]}$ selected cyanyellow (CY) space to realize the color eye image enhancement by saturation histogram equalization and luminance histogram correction. $\mathrm{Liu}^{[4]}$ accomplished the brightness and saturation enhancement for color X-ray image through consists sigmoid function and linear transformation function in hue-saturation-value (HSV) space. Whereas, all of these methods have a common characteristic that the color space must be appropriate to different enhanced purposes and methods, otherwise, the appearance of singular values in image pixel will influence the visual and enhanced effect.

Besides, Schaefer et al. ${ }^{[5]}$ realized the enhancement of the skin cancer color image through automatic color equalization $(\mathrm{ACE}) . \mathrm{Li}$ and Meng ${ }^{[6]}$ enhanced the color endoscope image by adaptive contrast diffusion. Zhang and Yang ${ }^{[7]}$ proposed a novel nonlinear gray transformation method to enhance the contrast of a typhoon cloud image. Guo et al. ${ }^{[8]}$ employed haziness analysis to realize the image processing. However, due to the large amount of calculation and parameter setting, most of the methods are limited in practical applications.

At the beginning of the 19th century, human visual system contains 3 kinds of color mechanisms was presented. In 1852, this theory has been supported by Helmholz, and that was the birth of the famous Young-Helmholz color vision theory. Cao and $\mathrm{Hao}^{[9]}$ put forward the bionic pattern recognition method based on Y-H model with ideal experiment results. Ding et al. ${ }^{[10]}$ proposed an image compression method by the singular value decomposition based on Y-H model. In 2013, the concept of "intensity numbers matrix histogram" and a kind of color medical image enhanced method based on color space transformation which are put 
forward in $[11,12]$. The color image can be enhanced without distortion by $\mathrm{Y}-\mathrm{H}$ transformation and equalization of "intensity numbers matrix histogram", and finally be displayed in the RGB color space by inverse Y-H transformation. In addition, the nonlinear extrapolation method ${ }^{[12]}$ and fuzzy set theory ${ }^{[13]}$ are combined with Y-H transformation for color image enhancement.

In order to achieve better enhanced effect, a new method consists of Y-H transformation and the adaptive equalization of intensity numbers matrix histogram will be proposed and elaborated in this paper.

The rest of this paper is organized as follows. Section 2 explains the Young-Helmholtz model which is the foundation of this research. In Section 3, the principle and flow of the new method is expounded in detail. Comparative experiments are conducted in Section 4 to verify the superiority of the new method. Finally, Section 5 gives the conclusion and future extension of this research.

\section{Young-Helmholtz model ${ }^{[11,12]}$}

A pixel of a human retina image can be expressed by the three-channel information of color image as

$$
f(x)=R \times 1+G \times \varepsilon+B \times \varepsilon^{2}
$$

where $\varepsilon^{3}=1$, and $1, \varepsilon, \varepsilon^{2}$ are hyper imaginary units; $f(x)$ is considered as the color numbers of pixel $x$ and defined by $Y$.

According to the Greaves color space transformation principle, three orthogonal axes $X, Y$ and $Z$ are corresponded to $R, G$ and $B$. A sphere is designed by setting the coordinate origin $O$ as the origin center, and these three points of intersection between the sphere and axes $X, Y, Z$ are denoted as $l, m, n$, respectively. The axis $e_{l u}$ is the vertical from the coordinate origin $O$ to the plane formed by points $l, m$ and $n$. The plane $E_{c h}$ passes through $O$ that is perpendicular to the axis $e_{l u}$. And then the color number $f$ can be expressed as

$$
f=a_{l u} e_{l u} \oplus Z_{c h} E_{c h}
$$

where $a_{l u}$ is the projection from $f$ to center axis $e_{l u}$, and $Z_{c h}$ is the perpendicular projection coordinate from $f$ to plane $E_{c h}$. Moreover,

$$
\begin{gathered}
a_{l u} \in \mathbf{R}, \quad Z_{c h} \in \mathbf{C} \\
e_{l u}:=\frac{1+\varepsilon+\varepsilon^{2}}{3}, E_{c h}:=\frac{\left(1+\omega_{3}^{2} \varepsilon+\omega_{3} \varepsilon^{2}\right)}{3}, \omega_{3}=: \mathrm{e}^{\frac{2 \pi i}{3}}
\end{gathered}
$$

where $e_{l u}$ and $E_{c h}$ are orthogonal, i.e., $e_{l u} E_{c h}=E_{c h} e_{l u}=$ $0, e_{l u}^{2}=e_{l u}$ and $E_{c h}{ }^{2}=E_{c h}$.

In (2), the real number $a_{l u}$ is the brightness (intensity) number, and the complex number $Z_{c h}$ is the chromaticity number. In the Y-H transformation method, the intensity numbers matrix is enhanced while the chromaticity numbers matrix remains unchanging, i.e., the color medical image can be enhanced without changing the correlation of
$R, G$ and $B$. This method can not only realize the image enhancement with low computational complexity, but also ensure the color's quality of the image after processing.

\section{Enhancement based on adaptive equalization of intensity numbers matrix histogram}

\section{1 "Intensity numbers matrix histogram" of the color image}

Reference [11] put forward the concept of intensity numbers matrix histogram of the color image for the first time, and the actual range of intensity numbers is confirmed as $0-\sqrt{255^{2} \times 3}, 0-442$ in rounding. The intensity numbers matrix histogram of the color image is also a discrete function, which can be expressed as

$$
g(k)=m_{k} k=0,1, \cdots, 442
$$

where $m_{k}$ is the number of pixel with the intensity level $k$ in $f(x, y)$.

\subsection{Adaptive equalization of intensity num- bers matrix histogram}

A method is similar to the local approach mentioned in $[14,15]$ that is adopted to process the intensity numbers matrix of images, and different contrast enhancement methods are used for different localities.

The formula of the local contrast enhanced method for calculating the intensity numbers matrix is expressed as ${ }^{[14]}$

$$
x_{i, j}^{\prime}=m_{i, j}+k\left(x_{i, j}-m_{i, j}\right)
$$

where $x_{i, j}$ and $x_{i, j}^{\prime}$ are the intensity levels before and after transforming respectively, $m_{i, j}=\frac{1}{m \times n} \sum_{(i, j) \in w} x_{i, j}$ is the average intensity level value of the pixel in window $W$.

In order to save the image details, the original image details are saved in advance, and added to the arithmetic during the histogram equalization process. Therefore, the histogram equalization arithmetic is modified as ${ }^{[15]}$

$$
x_{i, j}^{\prime}=\left\{\begin{array}{cl}
T\left(x_{i, j}\right)+k\left(x_{i, j}-m_{i, j}\right), & \text { if } 0 \leq x_{i, j} \leq 442 \\
T\left(x_{i, j}\right), & \text { otherwise }
\end{array}\right.
$$

where $m_{i, j}$ is the neighborhood average value of the window centered with $x_{i, j}, T$ is the transformation function of $x_{i, j}$. The criterion in (5) is set to ensure that the enhanced color image will not exceed the image intensity level range $[0,442]$. Equation (5) illustrates that $T$ plays the role of adjusting the dynamic range of the intensity numbers matrix histogram, and $k\left(x_{i, j}-m_{i, j}\right)$ plays the role of enhancing the local intensity level. In this process, the high-frequency noise is enhanced as well as the brightness of details. In order to avoid this phenomenon, the coefficient $k$ is adjusted 
adaptively, which can amplify the value of details and reduce the background noise.

The adaptive adjustment process of $k^{[16]}$ should meet the following conditions: when intensity level $x_{i, j}$ of the center pixel of window $W$ has no image detail, $k \rightarrow 0$; otherwise, $k \rightarrow$ larger value. $k$ can be expressed as the intensity level variance of window $W$ neighborhood, which is described as $^{[15]}$

$$
k=k^{\prime}\left(\frac{\sigma_{i, j}^{2}}{\sigma_{n}^{2}}-1\right)
$$

where $\sigma_{i, j}^{2}, \sigma_{n}^{2}$ are the intensity level, variance of window $W$ and the noise variance of whole image intensity level, respectively. $k^{\prime}$ is the proportional coefficient.

When $\sigma_{i, j}^{2}=\sigma_{n}^{2}$ and $k=0$, there is no image detail, and the intensity numbers matrix histogram enhancement is conducted on the intensity level of center pixel in the neighborhood. As $\frac{\sigma_{i, j}^{2}}{\sigma_{n}^{2}}$ grows, the value of $k$ becomes larger. When the side length $n$ is too small and the neighborhood variance $\sigma_{i, j}^{2}$ is sensitive to grain noise, the noise will be enhanced. Oppositely, when $n$ is too large, the noise which is in the detail instead of the detail neighborhood will also be enhanced. While the value of $k$ increases, the curve slope increases and the adaptive ability becomes stronger. However, if the value of $k$ is too large, the noise becomes more sensitive. This paper takes $(5 \times 5)$ domain with $k^{\prime}=0.0001$.

From all above, a mathematical expression of adaptive intensity numbers matrix histogram with complete detail enhancement is expressed as ${ }^{[16]}$

$$
x_{i, j}^{\prime}=\left\{\begin{array}{c}
T\left(x_{i, j}\right)+k\left(\frac{\sigma_{i, j}^{2}}{\sigma_{n}^{2}}-1\right)\left(x_{i, j}-m_{i, j}\right), \\
\text { if } 0 \leq x_{i, j} \leq 442 \\
T\left(x_{i, j}\right), \\
\text { otherwise. }
\end{array}\right.
$$

\subsection{Enhance process of color medical images}

The concrete steps of the enhancement based on adaptive equalization of intensity numbers matrix histogram is shown in Fig. 1.

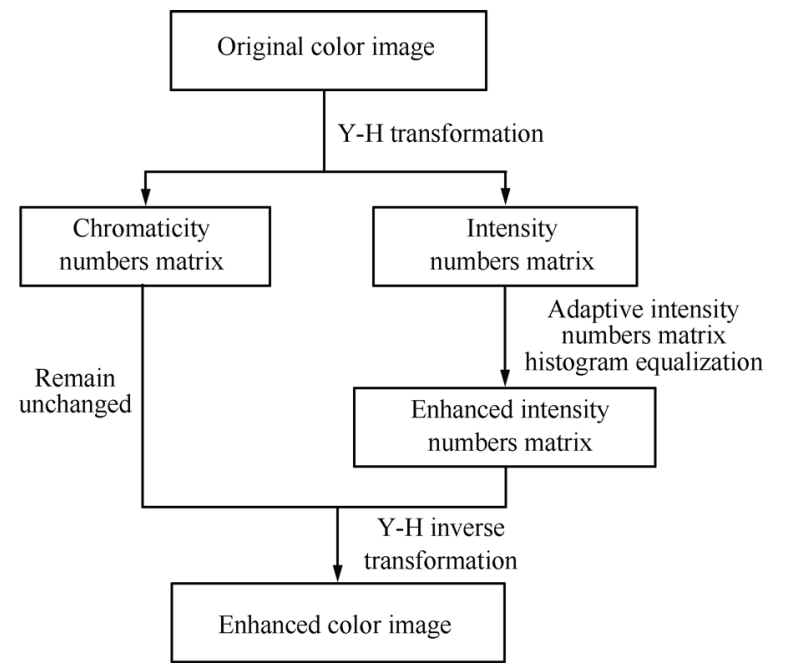

Fig. 1 Concrete steps of the method
Firstly, the intensity numbers matrix and the chromaticity numbers matrix of the original color image are obtained by $\mathrm{Y}-\mathrm{H}$ transformation. After given all the intensity levels $i$ and the numbers of each intensity level $n_{i}$, the intensity numbers matrix histogram and cumulative histogram of the original image can be calculated. Equation (7) is used to calculate the local intensity level and realize the equalization. Then, the new intensity numbers matrix histogram is obtained by $p\left(t_{i}\right)=\frac{n_{i}}{n}$. Finally, the enhanced image can be composed by the chromaticity numbers matrix which remains unchanging and the intensity numbers matrix after enhancing through inverse Y-H transform.

\section{Enhancement experiments and anal- ysis of the color medical images}

\subsection{Enhancement experiments}

The experiment research in image enhancement on four color pathological images is conducted in this paper, including renal cell carcinoma, squamous-cell carcinoma, chronic congestion of liver and colon polypoid adenomas. In order to prove the superiority of the method proposed in this paper, the enhancement results of the adaptive intensity numbers matrix histogram equalization method are compared with three common enhancement methods ${ }^{[17]}$, which are nonlinear transformation, multi-scale retinex algorithm (MSR) and guiding filter, as well as the intensity numbers matrix histogram equalization method mentioned in $[11,17]$. The original images are shown in Fig. 2, and the enhancement results of different methods are shown in Figs. 3-7.

\subsection{Analysis of the enhancement results}

Image quality mainly includes two aspects: the fidelity and the understandability of the image. From the subjective evaluation point of view, the image enhanced by the method in this paper has higher brightness without distortion, and its visual effect is better than general methods. But subjectively, it is difficult to distinguish the effect of the methods in [11] and this paper.

According to the objective evaluation method mentioned in [17], two indicators of the entropy and contrast improvement index (CII) are selected in this paper. Entropy (E) is a measure of the information amount. According to the theory, the image with higher entropy contains more information, and the image details are more abundant. It can be calculated by contrast improvement index defined by

$$
\begin{gathered}
\text { CII }=\frac{C_{\text {processed }}}{C_{\text {original }}} \\
\left\{\begin{array}{l}
E=-\sum_{i=1}^{M} \sum_{j=1}^{N} p_{i j} \ln p_{i j} \\
p_{i j}=\frac{f(i, j)}{\sum_{i=1}^{M} \sum_{j=1}^{N} f(i, j)} .
\end{array}\right.
\end{gathered}
$$



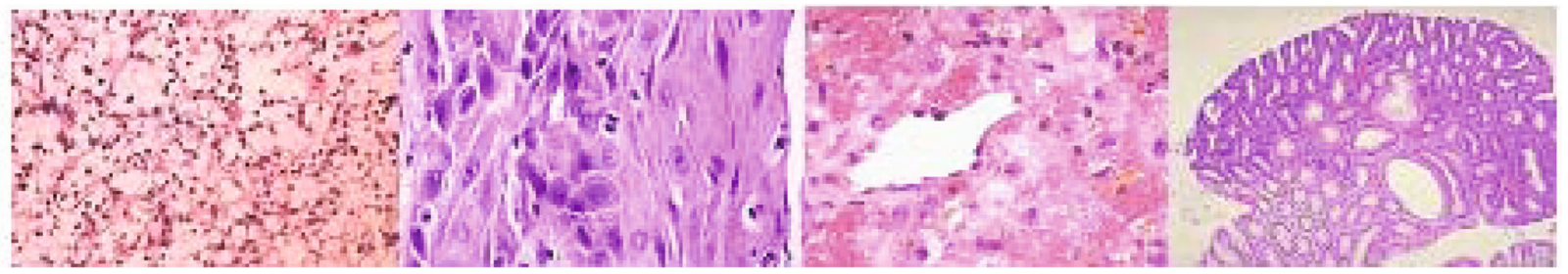

Fig. 2 Original color medical images
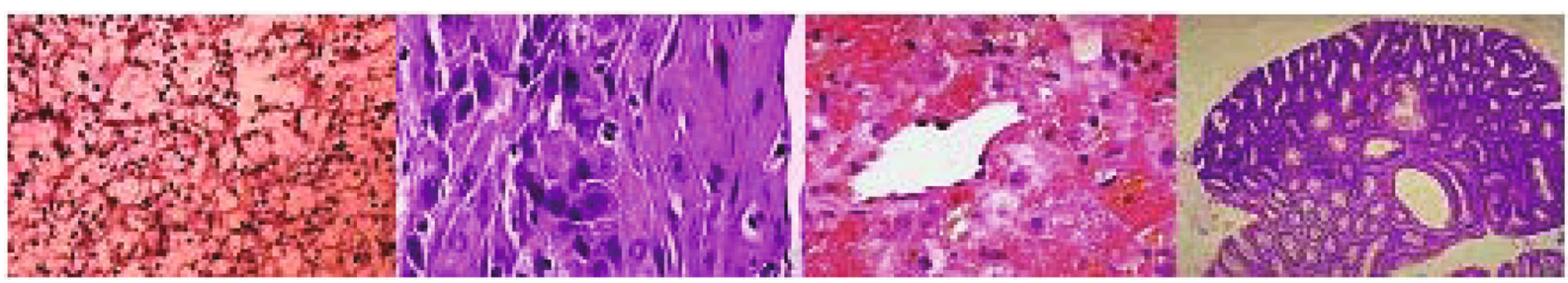

Fig. 3 Color medical images enhanced by nonlinear transform
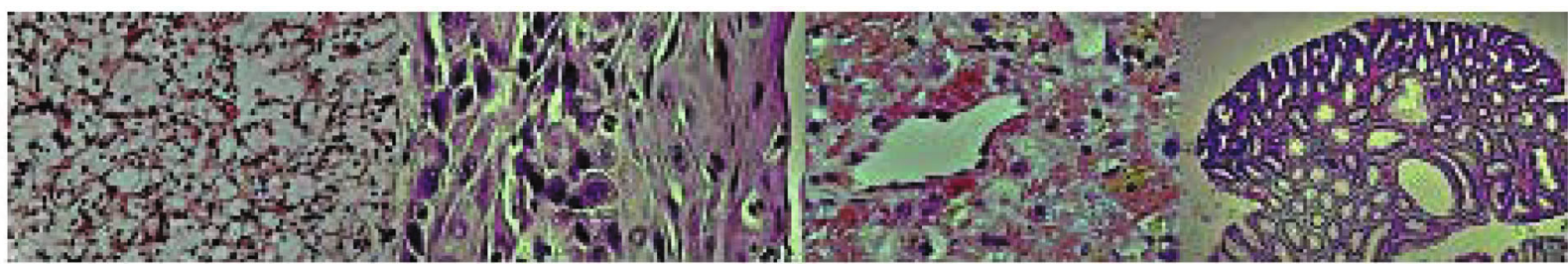

Fig. 4 Color medical images enhanced by MSR

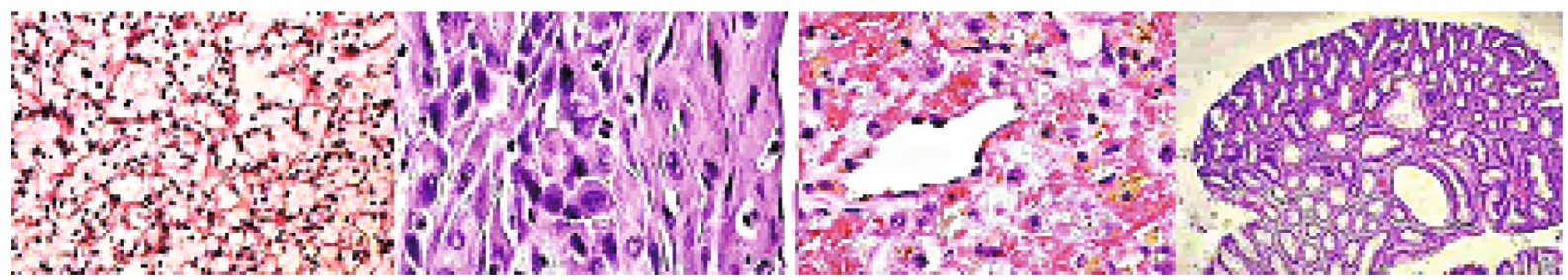

Fig. 5 Color medical images enhanced by guiding filter
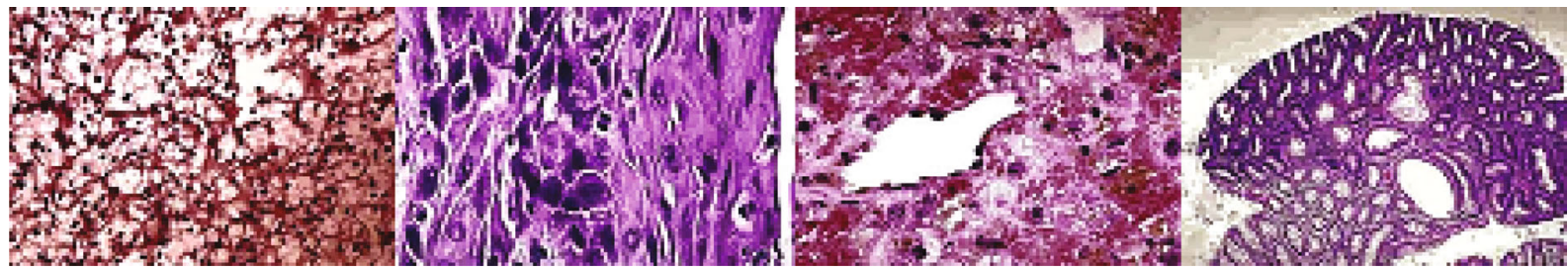

Fig. 6 Color medical images enhanced by intensity numbers matrix histogram equalization
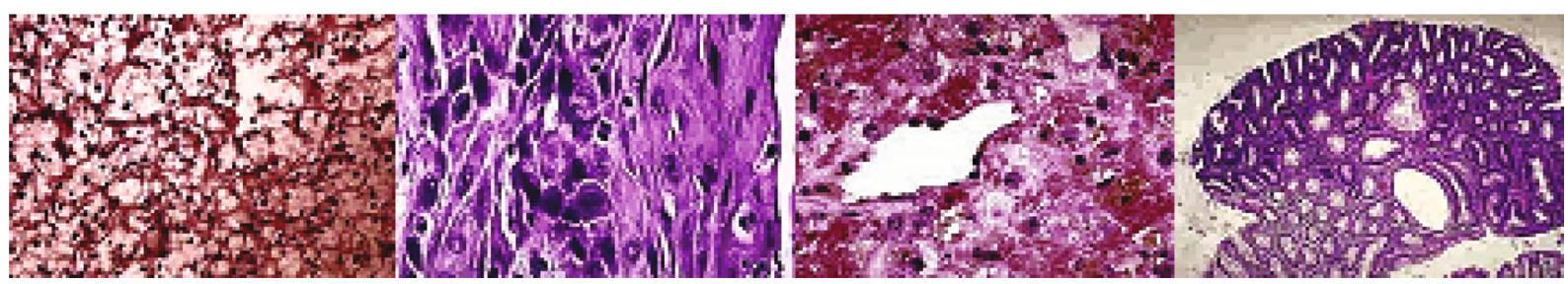

Fig. 7 Color medical images enhanced by adaptive intensity numbers matrix histogram equalization

CII is calculated as follows. The image (color image needs to be converted to a grayscale image) is divided into $3 \times 3$ small pieces. $C_{\text {processed }}$ is the average of all $3 \times 3$ small images' contrast of enhanced images. $C_{\text {original }}$ is the average of the corresponding original images. The contrast of 
each $3 \times 3$ small piece can be defined as

$$
\text { contrast }=\frac{\max -\min }{\max +\min }
$$

where max is the maximum gray value of the $3 \times 3$ small pieces, min is the minimum gray value. Subscripts original and processed are used to distinguish images before and after enhancement.

The images' entropies obtained by a variety of enhancement algorithms are shown in Table 1. The entropies of the original images and images enhanced by the nonlinear transformation, MSR, guiding filter and intensity numbers matrix histogram equalization are resulted from [17], which are compared with the results of adaptive intensity numbers matrix histogram equalization method in this paper. And the corresponding variation trends of the enhanced images' entropies are shown in Fig. 8.

The images' CII obtained by different enhanced algorithms are shown in Table 2. The CII of the original images and images enhanced by the nonlinear transformation, MSR, guiding filter and intensity numbers matrix histogram equalization are resulted from [17], which are compared with the results of adaptive intensity numbers matrix histogram equalization method in this paper. And the corresponding variation trends of the enhanced images' CII are shown in Fig. 9.

Tables 1 and 2 show that entropies of images, which are enhanced by adaptive histogram equalization method have increased. In other words, these images are enhanced by this method which contains more information. For CII of the enhanced images, this new method is superior to the traditional ones, like the nonlinear transformation, MSR and guiding filter. Except renal cell carcinoma images, the CII of other images keep almost unchanged or increased compared to these histogram equalization results in $[11,17]$.
Therefore, the contrast and visual effect of images is enhanced by the method in this paper.

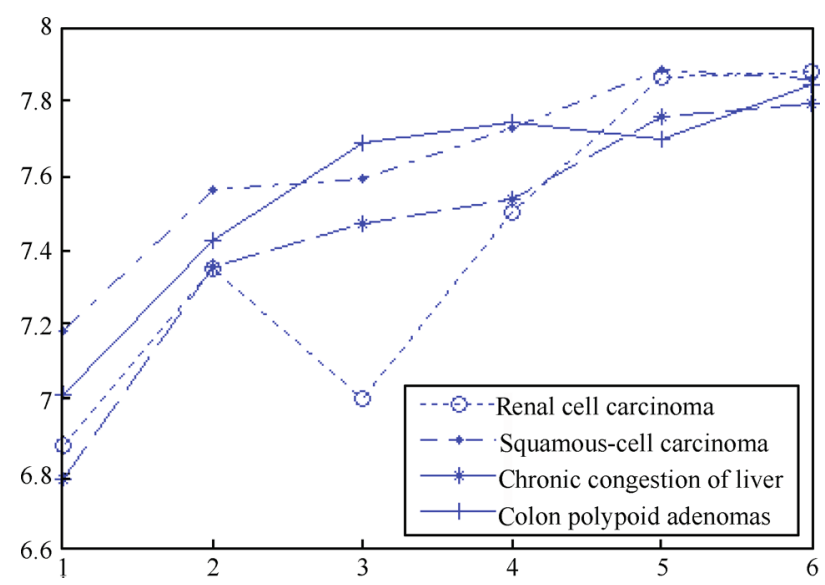

Fig. 8 Variation trends of the enhanced images' ${ }^{\prime}$ entropies

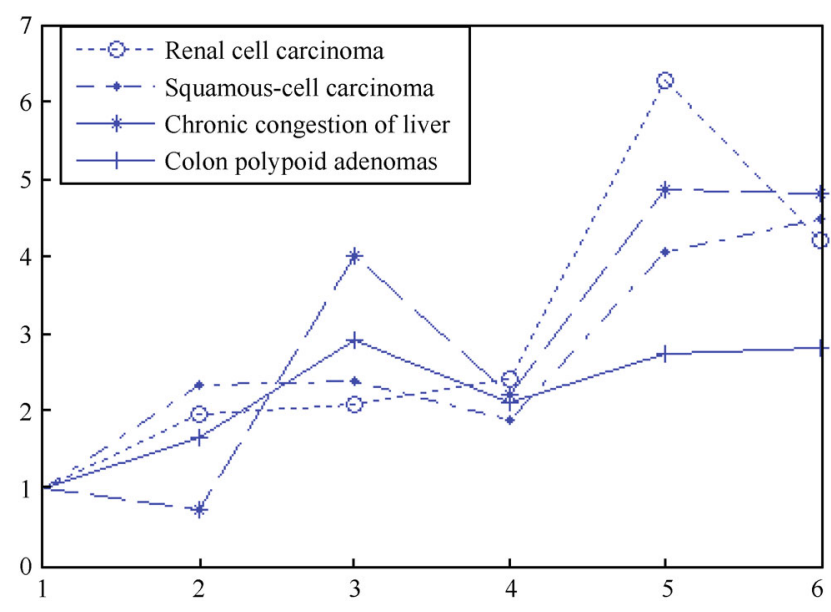

Fig. 9 Variation trends of the enhanced images' CII

Table 1 Entropies of the original and enhanced images

\begin{tabular}{cccccc}
\hline Images & Original image & Nonlinear transform & MSR & Guiding filter & Histogram equalization \\
& & & $\begin{array}{c}\text { Adaptive histogram } \\
\text { equalization }\end{array}$ \\
\hline Renal cell carcinoma & 6.8736 & 7.3530 & 6.9998 & 7.5001 & 7.8660 \\
Squamous-cell carcinoma & 7.1856 & 7.5633 & 7.5926 & 7.7282 & 7.8840 \\
Chronic congestion of liver & 6.7870 & 7.3575 & 7.4730 & 7.5369 & 7.7622 \\
Colon polypoid adenomas & 7.0140 & 7.4272 & 7.6901 & 7.7453 & 7.7009 \\
\hline
\end{tabular}

Table 2 CII of the original and enhanced images

\begin{tabular}{cccccc}
\hline Images & Original image & Nonlinear transform & MSR & Guiding filter & Histogram equalization \\
& & & $\begin{array}{c}\text { Adaptive histogram } \\
\text { equalization }\end{array}$ \\
\hline Renal cell carcinoma & 1.0000 & 1.9621 & 2.0887 & 2.4026 & 6.2900 \\
Squamous-cell carcinoma & 1.0000 & 2.3263 & 2.4005 & 1.8891 & 4.2000 \\
Chronic congestion of liver & 1.0000 & 0.7178 & 4.0886 & 2.2158 & 4.0602 \\
Colon polypoid adenomas & 1.0000 & 1.6441 & 2.9286 & 2.1173 & 4.8624 \\
\hline
\end{tabular}


On the other side, the enhancement effect can be also evaluated by the intensity numbers matrix histogram. The intensity numbers matrix histogram of the original images ${ }^{[17]}$, images enhanced by intensity numbers matrix histogram equalization ${ }^{[17]}$ and by adaptive intensity numbers matrix histogram equalization are shown in Figs. 10-13. It illustrates that the intensity grade distribution becomes wider and the distribution probability of the intensity grade in the low area becomes bigger, which means that the image's brightness has been enhanced and the image quality is improved.

\section{Conclusions}

A new enhancement method for color medical images based on Y-H transformation and adaptive intensity num- bers matrix histogram equalization has been designed and realized in this paper. This method can improve the brightness and contrast of images without changing the correlation of these three channels R, G and B. In terms of subjective evaluation, the enhanced color medical image displays without distortion and has higher brightness with better visual effect. On the objective evaluation aspect, the entropy and the CII of the image are increased significantly. "The intensity numbers matrix histogram" of color images illustrates that the intensity grade distribution becomes wider and the image quality has been obviously improved.

In the adaptive adjustment process of this method, the setting of parameters has a direct influence on the enhancement effect. Inappropriate choice of parameters may result in the increase of background noise, excessive enhancement, and decrease of detail information, which are the common
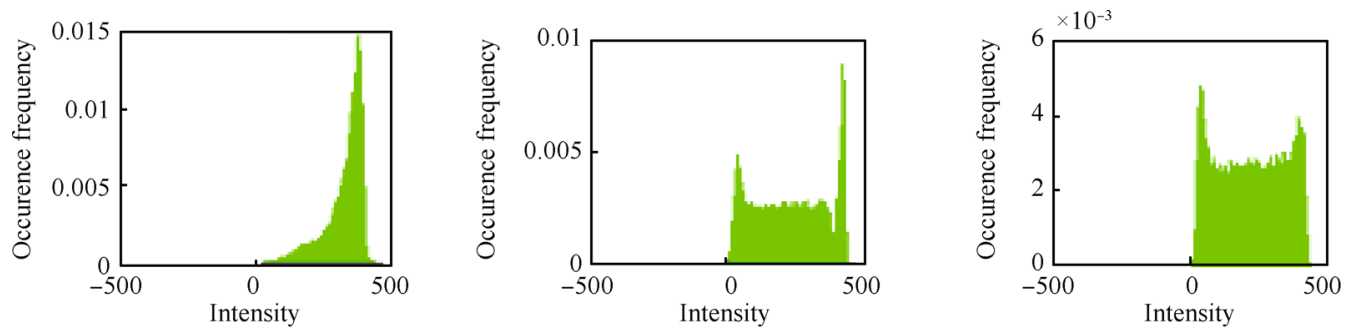

Fig. 10 The intensity numbers matrix histogram of the renal cell carcinoma image before and after enhancement
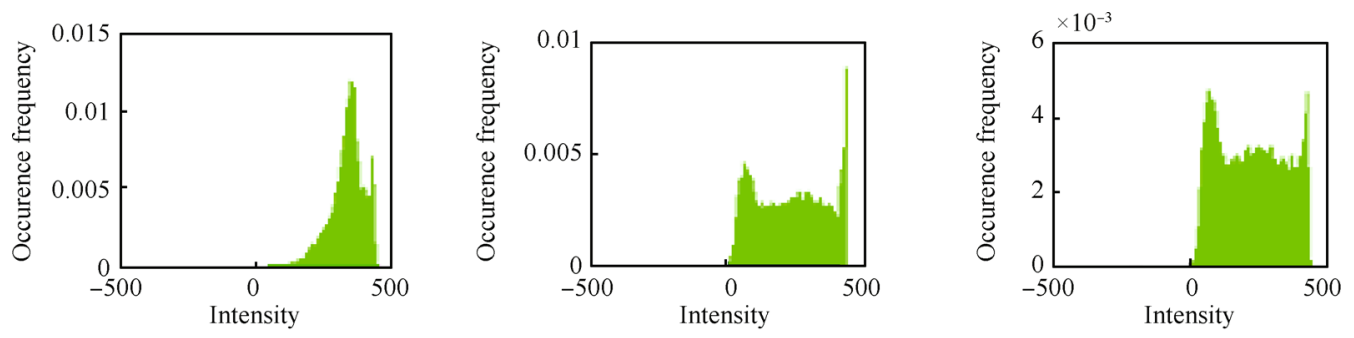

Fig. 11 The intensity numbers matrix histogram of the squamous-cell carcinoma image before and after enhancement
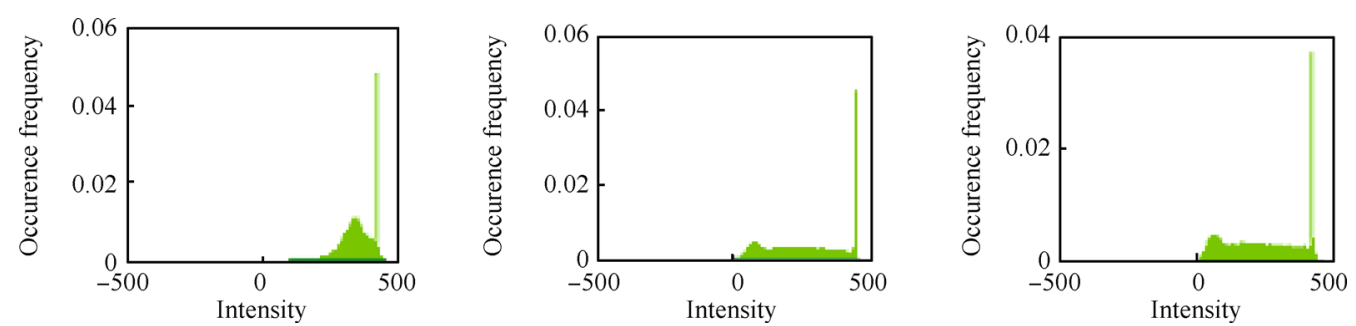

Fig. 12 The intensity numbers matrix histogram of the chronic congestion of liver image before and after enhancement
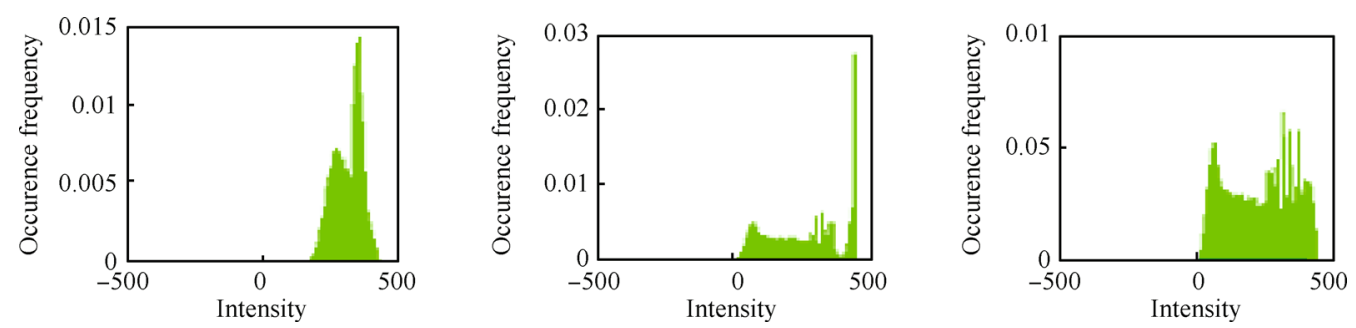

Fig. 13 The intensity numbers matrix histogram of the colon polypoid adenomas image before and after enhancement 
problems of the histogram transformation method. Therefore, on the basis of Y-H transformation theory, research on the enhancement method for medical images with wider application range is one of the future extensions. At the same time, the superiority of Y-H transformation theory has been verified in this paper, which provides the foundation for realizing the segmentation, compression and registration of images based on Y-H transformation in the future research.

\section{References}

[1] E. Blotta1, A. Bouchet, V. Ballarin1, J. Pastore. Enhancement of medical images in HSI color space. Journal of Physics: Conference Series, vol. 332, no. 1, pp. 12-41, 2011.

[2] Y. D. Yu, H. B. Xie, L. Qi. Study on the enhancement method of medical endoscope images. Chinese Journal of Biomedical Engineering, vol. 16, no. 1, pp. 31-34, 1997.

[3] X. W. Wang, L. S. Shen, D. H. Liu. A new algorithm for color medical image enhancement. Chinese Journal of Biomedical Engineering, vol. 23, no. 1, pp. 5-9, 4, 2004. (in Chinese)

[4] L. M. Liu. Research of color X-ray medical image enhancement in HSV space. Journal of Yunnan Normal University, vol. 31 , no. 5, pp. 56-60, 2011.

[5] G. Schaefer, M. I. Rajab, M. E. Celebi, H. Iyatomi. Colour and contrast enhancement for improved skin lesion segmentation. Computerized Medical Imaging and Graphics, vol. 35, no. 2, pp. 99-104, 2011.

[6] B. P. Li, Max Q. H. Meng. Wireless capsule endoscopy images enhancement via adaptive contrast diffusion. Journal of Visual Communication and Image Representation, vol. 23, no. 1, pp. 222-228, 2012.

[7] C. J. Zhang, B. Yang. A novel nonlinear algorithm for typhoon cloud image enhancement. International Journal of Automation and Computing, vol. 8, no. 2, pp. 161-169, 2011.

[8] F. Guo, J. Tang, Z. X. Cai. Image dehazing based on haziness analysis. International Journal of Automation and Computing, vol. 11, no. 1, pp. 78-86, 2014.

[9] W. M. Cao, F. Hao. Biomimetic pattern recognition based on the Young-helmholtz model of multispectral image. In Proceedings of the International Conference on Artificial Intelligence and Computational Intelligence, IEEE, Shanghai, China, vol. 11, pp. 398-402, 2009.

[10] L. J. Ding, L. Hua, H. Feng. Image compression method for singular value decomposition based on Y-H model. Computer Engineering, vol. 37, no. 23, pp. 203-204, 210, 2011.

[11] L. Hua, H. Feng, L. J. Ding, J. P. Gu, X. Wu. Method of color medical image enhancement based on color space conversion. International Journal of Digital Content Technology and Its Applications, vol. 7, no. 1, pp. 687-695, 2013.
[12] L. Hua, Z. T. Zhou, H. Feng, L. J. Ding. A new color medical image enhancement method. In IET International Conference on Information Science and Control Engineering, IEEE, Shenzhen, China, pp. 1-5, 2012.

[13] L. Hua, Z. T. Zhou, J. Yang, H. Feng, L. J. Ding, J. P. Gu. Fuzzy enhancement method for color medical images based on color space conversion. Applied Mechanics and Materials, vol. 380-384, pp. 3706-3709, 2013.

[14] J. S. Lee. Digital image enhancement and noise filtering by use of local statistics. IEEE Transactions on Pattern Analysis and Machine Intelligence, vol. PAMI-2, no. 2, pp. 165-168, 1980 .

[15] S. G. Wei. The Research of Medical Ultrasound Image Processing, Master dissertation, Harbin Engineering University, China, 2005. (in Chinese)

[16] G. Song, Y. H. Liu. A adaptive histogram equalization method with enhancing the details. Journal of Shandong University of Technology, vol. 29, no. 1, pp. 81-85, 1999. (in Chinese)

[17] Z. T. Zhou. Research on the Enhancement and Recognition Technology of Color Medical Image, Master dissertation, Nantong University, China, 2013. (in Chinese)

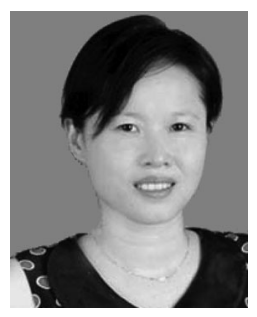

Ju-Ping Gu received the M.Sc. and Ph. D. degrees in electrical engineering from Southeast University, China in 1995 and 2003. She works in Department of Electrical Engineering, Nantong University, China. She has visited the Rheinisch-Westfaelische Technische Hochschule Aachen University (RWTH) in 2009 and now she is the dean of Department of Electrical Engineering at Nantong University, China. She has published more than 100 refereed journals and conference papers. She received the honorary titles such as Jiangsu Youth Awards, the Youth Science and Technology Leader of "333 High-level Personnel Training Project" of Jiangsu Province, Young and Middle-aged Academic Leader of the Provincial "Blue Project", Pacesetter of the Provincial Six Personnel Peak.

Her research interests include motors and control, robots and control, and signal processing.

E-mail: gu.jp@ntu.edu.cn (Corresponding author)

ORCID iD: 0000-0002-8769-5735

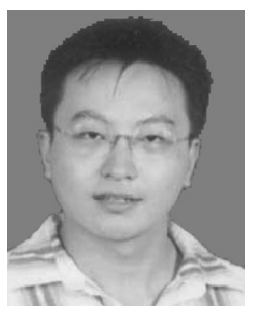

Liang Hua received the B. Eng. degree in industry automation industry from the Nantong Institute of Technology, China in 2001, and the M. Sc. degree in control theory and control engineering from Zhejiang University of Technology, China, in 2007. Since 2001, he works at Nantong University, China. Currently, he is an associate professor and deputy director of the labs in the Department of Electrical Engineering at Nantong University, China. He has published more than 40 refereed journals and conference papers. He received the honorary titles such as the Cultivation Object of " 333 High-level Personnel Training 
Project" of Jiangsu Province, Cultivation Object of the Provincial "Blue Project", and Outstanding Author of Academic Papers of Nantong in 2007, 2009 and 2011, respectively.

His research interests include motors and control, sensor and measurement control system and mode recognition.

E-mail: hualiang@ntu.edu.cn

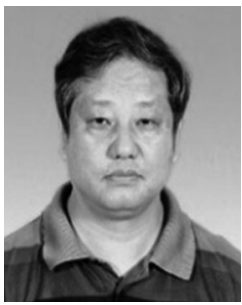

Xiao Wu received the B. Eng. degree in electrical engineering from Zhejiang University, China in 1982. He is the director of the labs in Department of Electrical Engineering at Nantong University, China. He has published more than 20 refereed journals and conference papers. He received the honorary titles such as the Advanced Educator of Nantong, Outstanding Communist and Virtue Pacesetter of Nantong University.

His research interests include automatic control, sensor and testing and intelligent building.

E-mail: wu.x@ntu.edu.cn

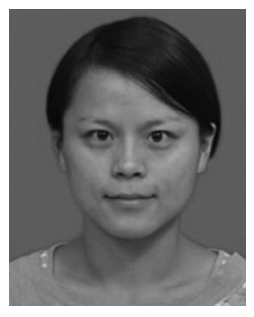

Hui Yang received the M.Sc. degree in control theory and control engineering from Nantong University, China in 2013. She works as an experimentalist in the labs of Department of Electrical Engineering at Nantong University, China.

Her research interest is mainly the signal analysis and processing.

E-mail: yanghui8828@ntu.edu.cn

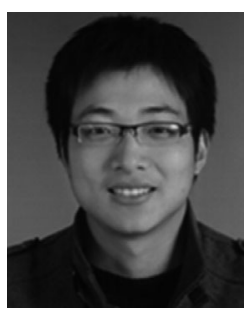

Zhen-Tao Zhou received the M.Sc. degree in control theory and control engineering from Nantong University, China in 2013.

His research interest is image processing. E-mail: zztntu@163.com 